\title{
Decompensated cirrhosis-related admissions in a large urban hospital in Uganda: prevalence, clinical and laboratory features and implications for planning patient management
}

\author{
*Apica BS, Ocama P, Seremba E, Opio KC, Kagimu MM \\ Department of Medicine, Mulago Hospital, Makerere University College of Health Sciences, Kampala, Uganda
}

\begin{abstract}
Background: Cirrhosis-related complications are a major cause of morbidity and mortality in areas where its risk factors are endemic.

Objective: We determined the prevalence of decompensated cirrhosis among patients on the gastroenterology service of Mulago Hospital and described the clinical and laboratory features of these patients.

Methods: All patients admitted to the unit were assessed and their diagnosis documented. Patients with cirrhosis had clinical features of decompensation recorded. History of alcohol consumption was taken and testing for hepatitis B surface antigen ( $\mathrm{HBsAg}$ ) and hepatitis $\mathrm{C}$ antibody (anti-HCV) performed.

Results: Between September 2010 and January 2011, we enrolled 482 patients. The majority (53.7\%) were male, overall median age 38 years. Decompensated cirrhosis was diagnosed in $85(17.6 \%)$ patients. Of the 85 patients, $47(55.3 \%)$ gave a history of alcohol intake, HBsAg was positive in $23(27.1 \%)$ and anti-HCV in $3(3.5 \%)$. Decompensation was defined by ascites among $81(95.3 \%)$ patients, variceal bleeding in 31 (36.5\%), encephalopathy in $20(23.5 \%)$.

Conclusion: Cirrhosis is common in Mulago hospital presenting mainly with ascites and variceal bleeding. Aside from controlling causes of liver diseases, especially alcohol and hepatitis B virus infection, in the interim it is necessary to manage complications in patients who already have cirrhosis.

African Health Sciences 2013; 13(4): 927 - 932 http://dx.doi.org/10.4314/ahs.v13i4.10
\end{abstract}

\section{Introduction}

Liver cirrhosis remains a major cause of morbidity and mortality worldwide ${ }^{1,2}$. Much of this morbidity and mortality is attributable to decompensation of liver function resulting in complications such as ascites, variceal bleeding and hepatic encephalopathy. Development of these complications leads to a poor quality of life consequent to repeated hospitalizations, increased cost of healthcare and marks the onset of a worsened prognosis ${ }^{3,4}$. Liver transplantation, the only curative treatment for cirrhosis, is not readily available because of a worldwide shortage of donor livers, logistical difficulties in the transplant programs and high costs of the procedure ${ }^{5}$. Prevention and management of these complications has therefore

\begin{tabular}{|l|}
\hline *Corresponding author: \\
Betty S. Apica \\
Department of Medicine \\
Mulago Hospital \\
Makerere University College of Health Sciences \\
P.O Box 7072 \\
Kampala, Uganda \\
Phone contact: +256772399846 \\
E-mail: bapica2k@yahoo.com \\
\hline
\end{tabular}

been a cornerstone of the management of patients with cirrhosis.

The prevalence of cirrhosis varies in different populations and is related to the existence of risk factors and their interaction. Liver cirrhosis is a common problem in Uganda because of high prevalence of risk factors. For example, the national prevalence of hepatitis B virus (HBV) infection in Uganda is $10 \%$ but varies from $3 \%$ to $25 \%$ depending on the region of origin in the Country ${ }^{6}$. At the same time excessive alcohol consumption is very common and Uganda was ranked the leading world consumer of alcohol by the World Health Organization $^{7}$ in 2004. Despite the high prevalence of risk factors for cirrhosis in Uganda, there is no data on the burden of decompensated cirrhosis in this setting to our knowledge.

The aim of this study was to determine the prevalence of liver cirrhosis and characteristics of decompensated cirrhosis in Mulago Hospital, Kampala in order to guide health interventions and allocation of resources for patient management. 


\section{Methods}

This was a cross sectional study in which patients admitted on the gastrointestinal and hepatology inpatient service of Mulago National Referral Hospital, Kampala, Uganda were recruited between September 2010 and January 2011. Patients were enrolled if they had a documented clinical diagnosis suggestive of a gastrointestinal or liver pathology. Socio-demographic data of patients and clinical data for the above diseases were recorded. Patients with suspected liver disease who had abdominal ultrasound characteristics of cirrhosis (nodular surface of the liver, heterogeneity of liver parenchyma or liver atrophy) and/or laboratory features consistent with cirrhosis (low serum albumin or low platelet count) were assessed for decompensation. Decompensation was defined by presence of ascites, hepatic encephalopathy or variceal bleeding. Ascites was graded as mild, moderate or severe based on the criteria of the international ascites club ${ }^{8}$. Hepatic encephalopathy was defined by detection of asterixis or using level of consciousness as described by Burroughs and graded thus: grade $1=$ confusion, grade $2=$ drowsiness, grade $3=$ stupor and grade $4=$ coma $^{9}$. Patients with asterixis only were classified as having grade 1 encephalopathy.

The presence of esophageal varices was confirmed by endoscopy in some patients who presented with upper gastrointestinal bleeding and graded according to the classification advocated by Beppu et al (grade $1=$ small and straight, grade $2=$ moderately sized, tortuous and occupying less than one third of the esophageal lumen or grade $3=$ large, coiled and occupying one third or more of the lumen $)^{10}$. In the remaining patients, abdominal collaterals with ascites plus hematemesis and or melena were construed as variceal bleeding. Physician diagnoses were initially recorded as free text then later coded. For example, dyspepsia and gastritis were grouped together and cholecystitis, cholelithiasis and cholangiocarcinoma were categorized as gall bladder disease. The primary diagnosis was used to group patients into the different categories.

In an attempt to establish the possible aetiology of cirrhosis and magnitude of decompensation of liver function, $10 \mathrm{mls}$ of blood was drawn and analyzed for liver function tests (Cobas Integra 400 plus, Roche, Switzerland), complete blood count (Beckman coulter Inc., France), HBsAg using HBsAg Dipstick (Cypress Diagnostics, Belgium) and anti-HCV using the One Step Anti-HCV Test, (Cypress Diagnostics, Belgium). Patients were considered to have HBV and Hepatitis $\mathrm{C}$ virus (HCV) infection if they tested positive for HBsAg and anti-HCV respectively.

History of alcohol consumption was taken from the patients. Because of widespread use of homebrewed alcohol and communal pattern of drinking in Uganda, it was difficult to quantify the amount of alcohol consumed by each participant so the CAGE score (Cutting down, Annoyed, Guilt, Eyeopener) for alcohol dependence was used as a surrogate measure of alcohol misuse.

Severity of liver cirrhosis was determined using Child Pugh score involving evaluation of 5 parameters including ascites, encephalopathy, serum bilirubin, serum albumin and International Normalized Ratio ${ }^{11}$.

The study was approved by the Institutional Review Board of Makerere University College of Health Sciences and all patients or their legal guardians consented to participate in the study. Children ( $N=20$, all between 13-17 years) were recruited after obtaining assent from them and consent from their legal guardians.

\section{Data analysis}

Data analyses were performed using Statistical Packages for Social Sciences 16.0 for Windows (SPSS Inc. Chicago, Illinois, USA). Continuous variables such as age, liver function tests and platelet counts were summarized as medians (interquartile range). Comparison between groups of categorical variables was performed using chi-square or Fisher's exact test. To test whether medians of selected continuous variables were meaningfully different, we used the Mann-Whitney's rank sum test. Statistical significance was defined as $\mathrm{P}$ value of $<0.05$.

\section{Results}

\section{Socio-demographic characteristics of the study population}

Four hundred and eighty two participants with gastrointestinal and hepatology-related illnesses were recruited. The majority of participants were young with median age of 38 years (IQR 28-54) and there was a slight male predominance $(53 \%)$. The sociodemographic characteristics of patients with decompensated cirrhosis were comparable to those with other gastrointestinal diagnoses (data not shown).

\section{Hospitalization for cirrhosis}

Eighty five patients $(17.6 \%)$ had decompensated cirrhosis, making this the leading single principal 
diagnosis on the gastrointestinal and hepatology service of the hospital. Dyspepsia 85 (17.6\%), liver cancer $65(13.5 \%)$ and diarrhoea $63(13.1 \%)$ were other major contributors to disease burden. This information is summarized in table 1.

Table 1: Clinical diagnoses on the gastrointestinal and hepatology inpatient service of Mulago Hospital, September 2010 to January $2011(\mathrm{~N}=482)$

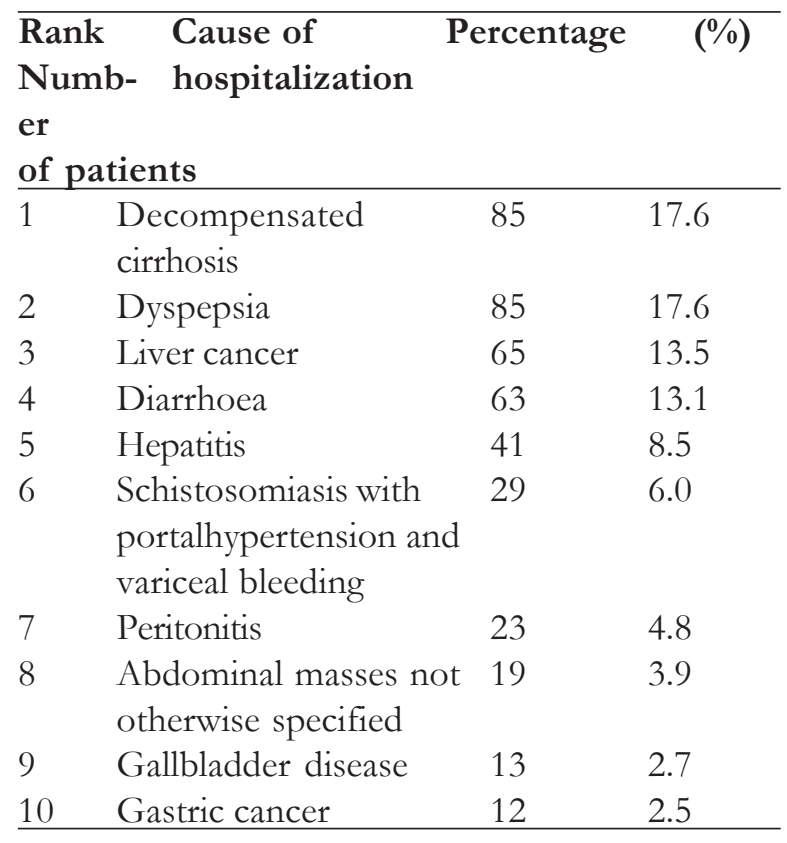

Presumed causes of cirrhosis

Of 85 patients with decompensated cirrhosis, 47 $(55.3 \%)$ gave a history of alcohol intake and 23 $(27.1 \%)$ tested positive for HBsAg (table 2). HCV infection was presumed to be the cause of cirrhosis in $3(3.5 \%)$ patients and no associated risks were identified in $23(27.1 \%)$. For those patients who gave a history of alcohol intake, the median duration of alcohol use was 15 years (IQR 10-28). CAGE score for alcohol dependence was positive in $17(36.2 \%)$ patients with history of alcohol use. Thirty $(63.8 \%)$ of these individuals met the liver enzyme criteria (aspartate aminotransferase:alanine aminotransferase $>2: 1)$ for defining alcohol related liver disease. Males with cirrhosis were more likely to have a history of alcohol intake than their female counterparts $(P=0.001$; odds ratio [OR], 4.5; 95\% Confidence Interval [CI]: $1.770-11.440$ ) but there was no significant association between gender and history of alcohol dependence $(P=0.464$; OR, 0.6; CI 0.152 2.371). The median duration of alcohol consumption was similar in males and females (median [IQR]: 15 [8.5-30] vs. $12[10-20]$ years $P=0.527)$. Half of the patients had been abstinent of alcohol for at least 6 months prior to admission and the main reason for this cessation was the onset of hepatic decompensation. Among patients with decompensated cirrhosis, more males $38.5 \%$ (20 of 52 ) tested positive for HBsAg compared to $9.1 \%$ (3 of 33) females $(P=0.003$; OR 6.25; CI 1.683-23.204). Conversely, females were less likely to have any of the three risk factors identified $45.5 \%$ (15 of 33) compared to males 90.4\% (47 of 52) ( $\mathrm{P}<0.001$; OR -11.28; CI 3.57635.579).

Thirteen patients (15.3\%) with cirrhosis tested positive for human immunodeficiency virus (HIV) infection and 3 of these patients were co-infected with HBV.

Table 2: Socio-demographic characteristics of patients with cirrhosis, associated factors, clinical and laboratory features of decompensated cirrhosis $(\mathbf{N}=85)$

\begin{tabular}{lc}
\hline Parameter & $\begin{array}{l}\text { Frequency (\%)/ } \\
\text { Median (IQR) }\end{array}$ \\
\hline Socio-demographic and risk factors \\
Age (years) & $42(32-55)$ \\
Male sex & $52(61.2 \%)$ \\
Alcohol use & $47(55.3 \%)$ \\
HBsAg positive & $23(27.1 \%)$ \\
HCV antibody positive & $3(3.5 \%)$ \\
HIV positive & $13(15.3 \%)$ \\
Clinical features & \\
Jaundice & $53(62.4 \%)$ \\
Lower limb oedema & $68(80 \%)$ \\
Collateral veins & $39(45.9 \%)$ \\
Splenomegaly & $37(43.5 \%)$ \\
Variceal bleeding & $31(36.5 \%)$ \\
Encephalopathy & \\
Grade 1 and 2 & $15(17.6 \%)$ \\
Grade 3 and 4 & $5(5.9 \%)$ \\
Ascites & $81(95.3 \%)$ \\
Child Pugh class & \\
B & $23(27.1 \%)$ \\
C & $6272.9 \%$ \\
Laboratory findings & \\
Alanine aminotransferase ( IU/L) & $28.8(21.1-$ \\
& $52.8)$ \\
Aspartate aminotransferase ( IU/L) $76.3(43.5-$ & $128.5)$ \\
Serum bilirubin (mg/dL) & $2.1(0.8-5.6)$ \\
Serum albumin $(\mathrm{g} / \mathrm{dL})$ & $2.1(1.7-2.5)$ \\
International Normalized Ratio & $2.1(1.6-2.8)$ \\
Prothrombin time (seconds) & $22.1(18.7-27.6)$ \\
Platelet count (x10 $/ \mu \mathrm{l})$ & $121(64-210)$ \\
Haemoglobin $(\mathrm{g} / \mathrm{dL})$ & $9.8(8.1-12.1)$ \\
\hline
\end{tabular}




\section{Complications of cirrhosis}

Ascites was the commonest complication that was documented among patients with decompensated cirrhosis, followed by variceal bleeding and hepatic encephalopathy, corresponding to $95.3 \%, 36.5 \%$ and $23.5 \%$, respectively (table 2 ). Forty $(47.1 \%$ ) patients had multiple complications of cirrhosis at the time of hospitalization. Ascites coexisting with variceal bleeding constituted the commonest combination of complications in $28(32.9 \%)$ patients. The largest proportion of patients had moderate and massive ascites seen in $38(44.7 \%)$ and $33(38.8 \%)$ patients respectively. Of 31 patients who presented with upper gastrointestinal bleeding, $7(22.6 \%)$ underwent endoscopy in which they were all confirmed to have esophageal varices. The varices were determined as grade 1 ( 3 of 7 ), grade 2 varices ( 2 of 7 ) and grade 3 varices ( 2 of 7$)$. The majority of study participants who presented with variceal bleeding had anaemia with median haemoglobin concentration of $8.9 \mathrm{~g} /$ dl (IQR 7.1-11.1 g/dl) and 58.6\% had thrombocytopenia with median platelet count of 120 $\times 10^{3} / \mu \mathrm{l}$ (IQR $\left.56.5 \times 10^{3}-190.5 \times 10^{3} / \mu \mathrm{l}\right)$. There was no significant difference in the median haemoglobin and median platelet counts between participants with variceal bleeding and participants without this complication $(P=0.152)$ and $(P=0.565)$ respectively. All participants with cirrhosis had low serum albumin (median $2.1 \mathrm{~g} / \mathrm{dL}$, IQR 1.7-2.5 g/dL).

Most of the patients with cirrhosis $(72.9 \%)$ had Child-Pugh class $C$ disease while 23 (27.1\%) had Child-Pugh class B.

\section{Discussion}

Cirrhosis represents an irreversible result of various insults to the liver that progress over decades and often remains clinically silent until decompensation occurs in the final stages. This dictates that management of this disease should focus on preventing progression and complications. In advanced stages of cirrhosis, liver transplant is the only curative treatment.

Our study highlights decompensated cirrhosis as the leading cause of admission to the gastrointestinal and hepatology service of this large tertiary care facility in Uganda. This is not surprising given the high prevalence of infectious and noninfectious causes of chronic liver diseases in this country. In this study, alcohol misuse and HBV infection, both of which are preventable, were the most frequently ascribed etiologies. These findings are in agreement with earlier studies that revealed high rates of alcohol consumption and a high HBV prevalence in the country ${ }^{6,7}$. Men had a significantly higher prevalence of alcohol-related cirrhosis compared to women. This disparity could be due to difference in drinking patterns between the two groups. One study from Uganda observed that a higher proportion of men consumed alcohol compared to women and men drank more frequently and took larger amounts compared to women $^{12}$. On the other hand, women could have had cirrhosis related to other causes that tend to have a female predilection such as primary biliary cirrhosis and chronic autoimmune hepatitis, but were not tested for in this study. Such findings have been seen in studies from the United States and Portugal which found alcoholic liver disease to be the main cause of death from chronic liver disease ${ }^{13,14}$. The authors from these papers also reported a significantly higher proportion of men with alcoholic liver cirrhosis compared to women. However, unlike in Uganda where chronic $\mathrm{HBV}$ is the second commonest cause of cirrhosis, chronic HCV ranks second in the United States and Europe. The proportion of HBV related cirrhosis may even be higher than apparent in this study because the rapid strip test used to diagnose HBV infection in this study has been shown to have low sensitivity of only $43.5 \%{ }^{15}$. On the other hand, $\mathrm{HCV}$ was not a major risk factor for cirrhosis $(3.5 \%)$ and this reflected the low prevalence of $\mathrm{HCV}$ in the general population. This could be due to less use of intravenous recreational drugs in Uganda.

Ascites was the leading complication present in $95.3 \%$ of patients and close to half of these patients also had another complication. The occurrence of multiple complications even further increases the morbidity in the affected patients. These findings are consistent with results of a study by Alvarez et al which found ascites to be the commonest decompensation and $46 \%$ patients presented more than one hepatic decompensation at admission ${ }^{16}$. Similarly Almani et al reported that ascites was the commonest complication occurring in $59 \%$ of patients with cirrhosis in Pakistan followed by hematemesis in $27 \%$ and hepatic encephalopathy in $9 \%{ }^{17}$. This trend of complications is similar to results of our study except the former study had smaller proportions of patients in each group given that they included patients with compensated cirrhosis.

Despite the large number of patients with variceal bleeding, endoscopy services were not readily 
accessible to these patients. Even then, the few patients who had endoscopy only received diagnostic services and not therapeutic endoscopic interventions. This underscores the need for improved endoscopic services so that patients with cirrhosis can receive standard treatment and surveillance for varices.

This study had some limitations. The diagnosis of cirrhosis was not confirmed by histology which could have led to erroneous diagnosis of cirrhosis. However, $71(83.5 \%)$ patients had abdominal ultrasound features consistent with cirrhosis. In the remaining 14 patients, clinical features of portal hypertension together with low albumin and/or thrombocytopenia were construed as cirrhosis. A study by Kryger et al ${ }^{18}$ showed clinical diagnosis of cirrhosis as an accurate method in a group of patients with alcoholic cirrhosis regardless of professional experience of the clinician. Similarly, some of the diagnoses other than cirrhosis were provisional working diagnoses made by gastroenterologists and no confirmatory tests had been performed in the study. This could have led to misclassification of the different disease categories. Failure to perform endoscopy on all patients to exclude other causes of gastrointestinal bleeding was another limitation of this study. In addition, asterixis, a non specific sign of liver disease was used as one of the signs of hepatic encephalopathy. Finally, the study did not investigate for some of the other important causes of cirrhosis such as autoimmune liver diseases, alpha-1-antitrypsin deficiency, hemochromatosis and Wilson's disease. It is therefore difficult to relate the few studied factors as the only causes of the cirrhosis.

\section{Conclusion}

Cirrhosis is common in Mulago Hospital, presenting mainly with ascites, variceal bleeding and encephalopathy. Aside from controlling causes of liver diseases, especially alcohol and HBV infection, in the interim it is necessary to manage complications in patients who already have cirrhosis.

\section{Acknowledgement}

This study was supported in part by the German Academic Exchange program (DAAD). The contents of the manuscript are the responsibility of the authors and do not necessarily represent the views of DAAD. The authors also acknowledge the patients who participated in the study as well as their families.

\section{References}

1. Blachier M, Leleu H, Peck-Radosavljevic M, Valla DC and Roudot-Thoraval F. The burden of liver disease in Europe. A review of available epidemiological data. European Association for the Study of the Liver. 2013; www.easl.eu/_eupolicy/eu-literature-review. Accessed 02 July 2013

2. World Health Organization. Age-standardized death rates: Liver cirrhosis by country. 2013; http:/ / a p p s.w ho. int / g ho/ d a t a / node. main.A1081?lang $=$ en? showonly $=$ GISAH. Accessed 02 July 2013

3. Björnsson E, Verbaan H, Oksanen A, Fryde'n A, Johansson J, Friberg $S$ et al. Health-related quality of life in patients with different stages of liver disease induced by hepatitis C. Scand J Gastroenterol 2009;44(7):878-87

4. Volk ML, Tocco RS, Bazick J, Rakoski MO and Lok AS. Hospital admissions among patients with decompensated cirrhosis. AM J Gastroenterol 2012;107(2):247-52

5. Van der Hilst CS, Ijtsma AJ, Slooff MJ, Tenvergert EM. Cost of liver transplantation: a systematic review and meta-analysis comparing the United States with other OECD countries. Med Care Res Rev. 2009;66:3-22.

6. Bwogi J, Braka F, Makumbi I, Mishra V, Bakamutumaho B, Nanyunja M et al. Hepatitis B infection is highly endemic in Uganda: findings from a national serosurvey. Afri Health Sci 2009;9(2):98-108

7. Global Status Report: Alcohol Policy, World Health Organization, Geneva, 2004

8. Moore KP, Wong F, Gines P, Bernardi M, Ochs A, Salerno F et al. The management of ascites in cirrhosis. Report on the concensus conference of the international ascites club. Hepatology 2003;38:258-266

9. Burroughs AK. Liver disorders -Management of chronic liver disease: In: Medicine International. 1990; Chapter 84:3472-3483.

10. Beppu K, Inokuchi K, Koyanagi N, Nakayama S, Sakata H, Kitano S et al. Prediction of variceal hemorrhage by esophageal endoscopy. Gastrointest Endosc 1981;27:213-218

11. Durand F and Valla D. Assessment of prognosis of cirrhosis. Semin Liver Dis. 2008;28(1):110-122

12. Tumwesigye NM and Kasirye R. Gender and the major consequences of alcohol consumption in Uganda. In: Obot IS, Room R, editors. Alcohol, gender and drinking problems: Perspectives from low and middle income 
countries; GENACIS Project. Geneva: World Health Organization; 2005. p. 189-208

13. Vong $\mathrm{S}$ and Bell BP. Chronic liver disease mortality in the United States, 1990-1998. Hepatology 2004;39:476-483

14. Cortez-Pinto H, Marques-Vidal P and Monteiro E. Liver disease-related admissions in Portugal: clinical and demographic pattern. Eur J Gastroenterol Hepatol 2004;16:873-877

15. Seremba E, Ocama P, Opio CK, Kagimu M, Yuan HJ, Attar N et al. Validity of the Rapid Strip Assay test for detecting HBsAg in patients admitted to hospital in Uganda. Journal of Medical Virology 2010;82:1334-1340
16. Alvarez MA, Cirera I, Sola R, Bargallo' A, Morillas RM and Planas R. Long-term clinical course of decompensated alcoholic cirrhosis. A prospective study of 165 patients. J Clin Gastroenterol 2011;45:906-911

17. Almani SA, Memon AS, Memon AL, Shah MI, Rahpoto MQ and Solangi R. Cirrhosis of liver: Etiological factors, complications and prognosis. JLUMHS 2008;61-66

18. Kryger P, Schlichting P, Dietrichson O and Juhl E. The accuracy of clinical diagnosis in acute hepatitis and alcoholic liver disease. Clinical versus morphological disease. Scand J Gastroenterol 1983;18: 691-696 